\title{
mAb 33 from Transduction Laboratories specifically binds to human CD95-L
}

\section{Dear Editor,}

Deregulated CD95-ligand (CD95-L/Fas-L/APO1-L) protein expression is suggested to be a major pathological mechanism underlying several disorders. Many of these CD95-L alterations were described based on the use of
A

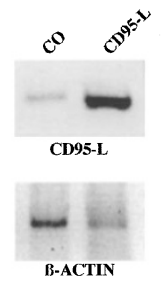

C

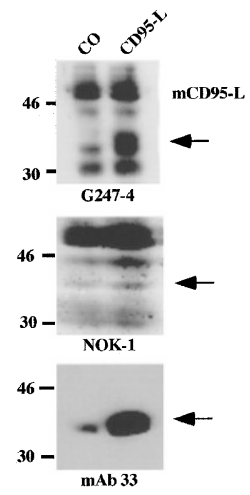

B

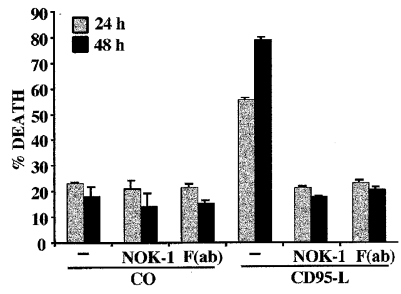

D

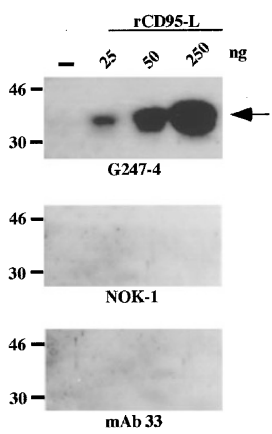

$\mathbf{F}$

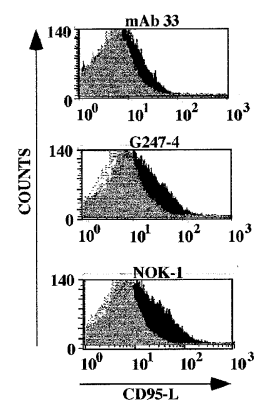

$\mathbf{E}$

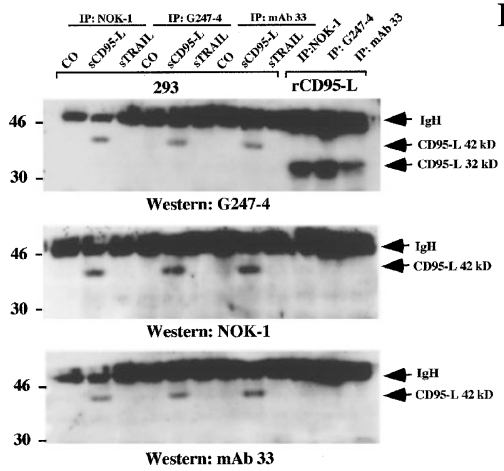

$\mathrm{mAb} 33$ from Transduction Laboratories (Lexington, KY, USA). Since the specifity of this antibody is unclear, ${ }^{1-3}$ we have established 293 cells stably transfected with vector encoding the full length human CD95-L cDNA. Overexpression of CD95-L mRNA was ensured by RT$\mathrm{PCR}^{4}$ (Figure 1A). Soluble CD95-L in supernatant of these cells induced death in target cells which was prevented by the CD95-L neutralizing mAb NOK-1 (Pharmingen, San Diego, CA, USA) or by blocking $\mathrm{F}(\mathrm{ab})_{2}$ anti-CD95 antibody fragments ${ }^{5}$ (Figure 1B). Using Western blotting, ${ }^{4} \mathrm{mAb} 33$ and other commercially available CD95-L monoclonal mouse IgG1 antibodies such as G247-4 (Pharmingen, San Diego, CA, USA) and NOK-1 yielded a band at the appropriate size of $37 \mathrm{kD}$ which was increased in CD95-L-transfected cells (Figure 1C). This suggests that CD95-L protein was specifically recognized by G247-4, NOK-1 and mAb 33 . However, while mAb 33 produced a single band, several bands were visible using G247-4 or NOK-1 which might be due to glycosylated forms ${ }^{6}$ or a high background binding. Another difference between these antibodies is their affinity to CD95-L protein which was highest for $\mathrm{mAb}$ $33(10 \mu \mathrm{g}$ protein loaded, $10 \mathrm{~s}$ exposure time), followed by NOK-1 (10 $\mu \mathrm{g}$ protein loaded, $5 \mathrm{~min}$ exposure time) and lowest for G247-4 (50 $\mu \mathrm{g}$ protein loaded, $45 \mathrm{~min}$ exposure time). Therefore, $\mathrm{mAb} 33$ is highly sensitive in a

predicted size of $37 \mathrm{kD}$ for CD95-L is marked by arrows. Ponceau red staining confirmed equal protein loading. (D) Recombinant CD95-L protein (rCD95-L) from Alexis was detected by Western blot analysis. The predicted size of 32 to $35 \mathrm{kD}$ for $\mathrm{rCD} 95-\mathrm{L}$ is marked by an arrow. (E) For immunoprecipitation of CD95-L supernatants from 293 cells stably overexpressing empty vector or the cDNAs for CD95-L or TRAIL as well as rCD95-L (Alexis) diluted in medium were analyzed by immunoprecipitation with either NOK-1, G247-4 or mAb 33 together with protein A sepharose. Equal parts of each immunoprecipitate were examined in three different Western blot experiments using either G2474 , NOK-1 or mAb 33. The specific bands for soluble CD95-L or the heavy chain of the antibody $(\mathrm{IgH})$ are marked by arrows. The size of a molecular weight marker in $\mathrm{kD}$ is indicated on the left. (F) Surface expression of CD95-L protein in stably transfected 293 cells was determined using mAb 33, G247-4, NOK-1 or isotype IgG1 control mAb by FACS-analysis and a representative out of five experiments is shown. CD95-L staining is represented by black profiles, empty vector staining by grey profiles and staining controls are marked by dotted lines. Prior to measurement of surface CD95-L expression by FACS-analysis cells were incubated for $12 \mathrm{~h}$ with $10^{-3} \mathrm{mM}$ of the CD95-L specific metalloproteinase inhibitor KB8401 (Pharmingen, Hamburg, Germany). After harvesting, cells were stained with $10 \mu \mathrm{g} / \mathrm{ml}$ of either mAb 33, G247-4, NOK-1 or IgG1 isotype control mAb (Becton Dickinson, Heidelberg, Germany) followed by labeling with $20 \mu \mathrm{g} / \mathrm{ml} \mathrm{PE-coupled} \mathrm{goat} \mathrm{anti-mouse} \mathrm{secondary} \mathrm{Ab}$ (Immunotech, Hamburg, Germany). Fixed cells were analyzed on a FACScan flow cytometer using the Cell Quest software (Becton Dickinson). An ELISA plate coated with immunogens at $2 \mu \mathrm{g} / \mathrm{ml}$ was incubated with dilutions of $\mathrm{mAb}$ 33 starting at $1 \mu \mathrm{g} / \mathrm{ml}$ using a standard protocol 
Western blot assay and loading too much protein extract may saturate the ECL-reaction masking differences between low or high level expression of CD95-L., ${ }^{1,2}$ To further characterize G247-4, NOK-1 and mAb 33, the binding properties to recombinant CD95-L (rCD95-L) from Alexis (Grünberg, Germany) were evaluated. rCD95-L corresponds to the extracellular domain of human CD95$\mathrm{L}$ and is fused at the $\mathrm{N}$-terminus to a linker peptide and a tag. After SDS-PAGE and Western blotting, a specific band with a proposed molecular weight of 32 to $35 \mathrm{kD}$ was detected by G247-4 (Figure 1D). Neither NOK-1 nor $\mathrm{mAb} 33$ stained $\mathrm{rCD} 95-\mathrm{L}$ even after $1 \mathrm{~h}$ of exposure following the ECL reaction. On the other hand, NOK-1 completely neutralizes the cytotoxic activity of rCD95-L derived from Alexis (not shown) suggesting that NOK-1 specifically recognizes CD95-L. This means that the reducing conditions of the Western blot experiment together with the linker sequences of the recombinant protein may alter some CD95-L epitopes.

To compare the binding capacities of G247-4, NOK-1 and $\mathrm{mAb} 33$ under non-reducing and reducing conditions we performed an immunoprecipitation assay using supernatants from 293 cells and rCD95-L from Alexis. Supernatants or $\mathrm{rCD} 95-\mathrm{L}$ in cell culture medium were incubated with G247-4, NOK-1 or mAb 33 under non-reducing conditions followed by precipitation with Protein $A$ sepharose (Sigma, Deisenhofen, Germany). The immunoprecipitates were divided into three equal portions and each of them was analyzed by Western blotting. Probing the membranes with either G247-4, NOK-1 or mAb 33 detected enhanced levels of soluble CD95-L protein in CD95-L-transfected 293 cells only (Figure 1E). Soluble CD95-L migrated at a molecular weight of about $42 \mathrm{kD}$ which correspond to a glycosylated form. ${ }^{6}$ Thus, full length CD95-L lacking any linker sequences is detected under non-reducing and reducing conditions by G247-4, NOK-1 and $\mathrm{mAb}$ 33. Truncated CD95-L protein with linker sequences (Alexis) was also specifically bound by G2474 , NOK-1 or mAb 33 under the non-reducing immunopre- cipitation conditions since G247-4 stained a band with the proposed molecular weight. In contrast, neither NOK-1 nor mAb 33 detected immunoprecipitated rCD95-L (Alexis) after Western blotting. These data clearly demonstrate that under reducing conditions linker peptide and tag of rCD95-L mask epitopes which are required for the binding of NOK-1 and mAb 33.

To investigate the functionality of $\mathrm{mAb} 33, \mathrm{G} 247-4$ and NOK-1 in FACS analysis surface expression of CD95-L in the transfected 293 cells was examined. A species- and isotope-matched antibody was used as control. All of the CD95- $L$ antibodies gave an enhanced staining in CD95- $L$ overexpressing cells although with different affinities since the shift was highest using NOK-1 and lowest using mAb 33 (Figure 1F).

On the basis of these results we conclude that mAb 33 is suited for analyzing natural CD95-L protein by Western blot an immunoprecipitation. $\mathrm{mAb} 33$ is less sensitive in detecting surface expression CD95-L by flow cytometry. $\mathrm{mAb} 33$ specifically binds rCD95-L from Alexis only in its native form e.g. in immunoprecipitation or neutralization assays but not in the denatured form e.g. in Western blot analysis.

\footnotetext{
I Herr ${ }^{* 1}$, C Posovsky ${ }^{1}$, T Böhler ${ }^{2}$ and K-M Debatin ${ }^{2}$

${ }^{1}$ Deutsches Krebsforschungszentrum, Pediatric Oncology - D0800, Im Neuenheimer Feld 280, 69120 Heidelberg, Germany; ${ }^{2}$ Universitäts Kinderklinik, Prittwitzstraße 43, 89075 Ulm, Germany

*Corresponding author: I Herr, Deutsches Krebsforschungszentrum, Pediatric Oncology - D0800, Im Neuenheimer Feld 280, 69120 Heidelberg, Germany. Tel: +49-6221-42-3366; Fax +49-6221-42-3362;

E-mail: i.herr@dkfz.heidelberg.de
}

1. Stokes TA et al. (1998) Science 279: 2015
2. Fiedler P et al. (1998) Science 279: 2015
3. Papoff G et al. (1998) Science 279: 2015
4. Herr I et al. (1997) EMBO J. 16: 6200
5. Dhein J et al. (1995) Nature 373: 438
6. Tanaka M et al. (1995) EMBO J. 14: 1129 